\title{
SARS-CoV-2 Infection in Intensive Care Unit Healthcare Workers in Turkey: A Tertiary Center Cohort Study
}

\author{
๑ Sinan Uzman, ๑ Tulin Akarsu Ayazoglu* \\ University of Health Sciences Turkey, Haseki Training and Research Hospital, Clinic of Anesthesiology and Reanimation, Istanbul, Turkey \\ *Medeniyet University Faculty of Medicine, Department of Anesthesiology and Reanimation, Istanbul, Turkey
}

\section{Abstract}

\begin{abstract}
Aim: Intensive care unit (ICU) workers have a greater risk of being infected due to procedures requiring intensive contact with the coronavirus disease-2019 (COVID-19) patient. We aimed to explain how we applied the protection of healthcare workers against the contagion of COVID-19 infection in the ICU of a university hospital.

Methods: The study was performed in ICUs of the Goztepe Training Research Hospital with 650 beds which was affiliated to Medeniyet University in Istanbul, Turkey. We recorded the number of COVID-19 patients. We also recorded ICU workers (doctor, nurse, and others), and their working layouts. The frequency of the contagion of severe acute respiratory syndrome coronavirus-2 (SARS-CoV-2) to ICU workers was investigated. We also evaluated precautions for the prevention and evaluated the arrangement of ICUs for COVID-19 patients.
\end{abstract}

Results: Between March $17^{\text {th }} 2020$ and May $26^{\text {th }} 2020$ totaly, a total of 120 hospitalized COVID-19 patients were treated in ICUs. Laboratory confirmed COVID-19 was detected in only one health-care worker in ICUs.

Conclusion: Our experience showed that the correct and proper use of personal protective equipment according to the protocols are effective in protecting ICU workers against the contagion of SARS-CoV-2.

Keywords: COVID-19, SARS-CoV-2, intensive care unit, health-care workers, personal protective equipment

\section{Introduction}

Since the first case of coronavirus disease-2019 (COVID-19) was identified in Istanbul city of Turkey on March $9^{\text {th }}, 2020$, healthcare workers have been battling against the COVID-19 caused by a new type of coronavirus, severe acute respiratory syndrome coronavirus 2 (SARSCoV-2) (1). After which despite all the efforts, the number of cases increased exponentially and more than one hundred fifty thousand new cases of COVID-19 were diagnosed until May $19^{\text {th }}$. Besides that Ministry of Health has been reported that 4199 patients died due to this infection as of May 19th in Turkey (Figure 1).

Healthcare workers are at high risk for the contagion of COVID-19 infection. The National Health Commission of China has been reported that 1,716 (3.8\% of laboratoryconfirmed cases) health-care workers had been infected with the COVID-19 infection on February 14 2020 (2). The number of infected health-care workers increased rapidly to 3387, as of 25 February 2020 (3). As of April $30^{\text {th }}$, The Turkish Minister of Health announced that the number of infected health workers was 7428 (6.18\% of laboratory-confirmed cases) (4).

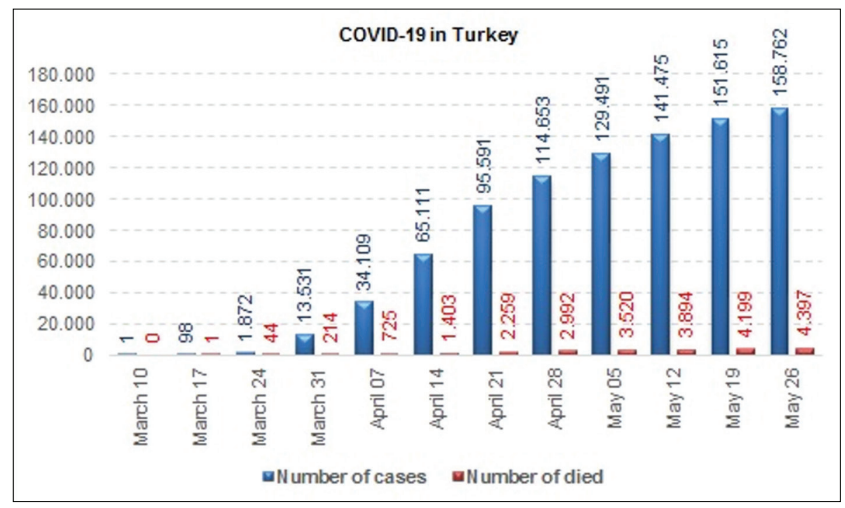

Figure 1. COVID-19 in Turkey

COVID-19: Coronavirus disease-2019

Address for Correspondence: Sinan Uzman, University of Health Sciences Turkey, Haseki Training and Research Hospital, Clinic of Anesthesiology and Reanimation, Istanbul, Turkey Phone: +90 5055645271 E-mail: drsinanuzman@yahoo.com ORCID: orcid.org/0000-0003-3166-7302 Received: 01.12.2020 Accepted: 28.01.2021

'Copyright 2021 by The Medical Bulletin of istanbul Haseki Training and Research Hospital The Medical Bulletin of Haseki published by Galenos Yayınevi. 
Although there are official recommendations from the World Health Organization (WHO) and other national and international communities to protect healthcare workers from this infection, the facilities and resources required for their implementation may not be sufficient in all centers dealing with the treatment of SARS-CoV-2 infection.

Especially ICU workers are at a greater risk of being infected due to procedures requiring intensive contact such as respiratory therapy, vascular and urinary catheterization and, nursing care with the COVID-19 patient. It has been reported that severe pneumonia with progressive hypoxemia requiring mechanical ventilatory support in the ICU may occur during the course of COVID-19 approximately in $12-17 \%$ of patients $(5,6)$. Thereby, in this article, we aimed to explain how we applied the protection of healthcare workers against the contagion of COVID-19 infection in the ICU and to identify healthcare workers infected with COVID-19.

\section{Methods}

\section{Study Design}

The study protocol was approved by the local hospital ethics committee (date/number: June $3^{\text {th }}$ 2020/0325) and conducted according to the Declaration of Helsinki. The study was performed in the ICUs of the Goztepe Training and Research Hospital with 650 beds which were affiliated to Medeniyet University in Istanbul, Turkey. Before the WHO declared a pandemic on March $11^{\text {th }}$, 2020, arrangements were performed in the hospital and the admission and triage protocols were established for the confirmed or suspected COVID-19 patients according to the Republic of Turkey Ministry of Health COVID-19 guidelines.

\section{Statistical Analysis}

A hospital audit database was used to obtain study data. Data extracted from the audit database were provided using hospital computer software (Nucleus, Monad software, Cankaya, Ankara, Turkey). We recorded the number of COVID-19 patients. We also recorded the ICU workers (physician, nurse, and others) and their working orders. We investigated the frequency of contagion of SARS-CoV-2 to ICU workers in the ICUs and Intermediate Care Unit (IMCU). Also, we evaluated precautions for the prevention and evaluated the arrangement of ICUs for COVID-19 patients.

Intensive Care Unit (ICU) admissions are dependent on the severity of the infection and in accordance with the established criteria such as requiring invasive respiratory support, hemodynamic support with vasoactive drugs, the patients were admitted to our two ICUs with 8 (ICU-I) and 6 (ICU-II) beds. ICU-I and II were on the first and second floor of the emergency building respectively. In addition, a 5 -room service ( 1 bed in each room) was created on the same floor with the ICU-I for the patients who received high-flow nasal cannula (HFCN) oxygen therapy IMCU. Thus, this area was separated from the other parts of the hospital.

\section{Results}

Between March $17^{\text {th }} 2020$ and May $26^{\text {th }} 2020$ a total of 120 of 871 hospitalized COVID-19 patients were treated in ICUs and IMCU. Laboratory confirmed COVID-19 was detected in only one health-care worker in ICUs from the admission of the first case to the ICU on March $17^{\text {th }}, 2020$ until May $26^{\text {th }} 2020$.

A shift work scheduled was prepared for 21 anesthesiologists, 25 residents (21 anesthesiology and 4 internal medicine), 47 nurses, and 19 other personnel (totally 112). ICUs and IMCU were managed by two anesthesiology and reanimation specialist who has an academic degree (associate professor) from Monday until Friday between 08 am and 16 pm during the study period. One of the remaining 19 anesthesiologists worked in shifts (16 pm-08 am from Monday to Friday and 24 hours on the weekend). In each of ICU-I and ICU-II two anesthesia residents and, in the IMCU one of the internal medicine residents served for 24 hours. Sixteen or 24-hour shifts were set up for nurses and other personnel. In each shift, 4 nurses were assigned for ICU-I and, 3 for ICU-II and IMCU. All doctors and nurses were on leave for two or three days after the shift.

Nasopharyngeal and throat swabs were obtained from all ICU workers to perform reverse transcriptionpolymerase chain reaction (RT-PCR) analysis for SARS-CoV-2 at least once regardless of the presence of symptoms. COVID-19 IgM and IgG dual antibody (Colloidal Gold) rapid test kit (Guanzhou Weimi Bio-Tech Co., Ltd., China) for finger-prick blood samples was used as a screening test for COVID-19. On April 17 $7^{\text {th }}$, a nurse suffered from fever, sore throat, and cough while on leave and she was diagnosed with COVID-19 confirmed by RT-PCR. She had no respiratory distress and posteroanterior chest radiography was normal. She was isolated at home for 15 days and successfully treated with hydroxychloroquine (2x400 mg loading dose and 2x200 mg for five days) and azithromycin (first day 500 mg, then four days $250 \mathrm{mg}$ ).

\section{Discussion}

Laboratory confirmed (RT-PCR) COVID-19 was observed in only one of $112(0.09 \%)$ ICU workers. How did we manage to minimize the number of infected ICU workers? For this purpose, we offer the following precautionary 
suggestions for the ICU procedures based on available evidence. Furthermore, we share our domestic experiences.

\section{Training About COVID-19}

While our first COVID-19 patient has not yet been admitted to the ICU, after the WHO declared a pandemic on March $11^{\text {th }}$, ICU workers consisting of doctors, nurses, and cleaning staff have been trained about the upcoming process by the infection control committee of our hospital. Insufficient attention to personal protection measures at the beginning of the epidemic due to lack of knowledge in Wuhan was the most important reason why healthcare workers get infected (7). It has been reported that the presence of health workers who died or got sick due to COVID-19 and, rumors and misinformation led to an increase in the level of anxiety in healthcare workers and a decrease in the will to work (8). Informing and educating of healthcare professionals about the disease and process was important in terms of applying personal protective measures, relieving their anxiety, and ensuring their motivation.

\section{Precautions of Prevention During the Procedures for Respiratory Support}

The contagion of the SARS-COV-2 occurs mainly through respiratory droplets and close contact. Therapeutic interventions for respiratory support including ambu/ mask ventilation, endotracheal intubation, non-invasive mechanical ventilation, high-flow nasal catheter therapy, bronchoscopy, and endotracheal suction are aerosolgenerating procedures and have a high risk of virus transmission as it increases the risk of direct contact with the droplet $(9,10)$. Personal protective equipment (PPE) (Special work clothes, surgical bonnet, FFP2/N95, latex glove, goggles, face shield, protective clothing, and rubber boot) was used to minimize transmission and it was always available in sufficient numbers. Donning and removing of PPE to manage COVID-19 patients is performed carefully in accordance with the protocols (11).

\section{Endotracheal Intubation}

We tried to perform endotracheal intubation via a video-guided laryngoscope under elective conditions as much as possible. We developed a transparent fiberglass protective box for tracheal intubation (Figure 2). The patient's airway was evaluated for difficult intubation. We applied preoxygenation for 3 minutes with 100\% oxygen. Then we used midazolam 2-3 mg plus fentanyl 50-100 $\mu \mathrm{gr}$ or ketamin $1-2 \mathrm{mg} / \mathrm{kg}$ and high doses of rocuronium $(1.5 \mathrm{mg} / \mathrm{kg})$ to ensure sedation and muscle relaxation. We performed rapid intubation within 50 seconds following low-pressure mask ventilation. Endotracheal intubations are performed by the most experienced anesthesiologist. Our results showed that all of the endotracheal intubations

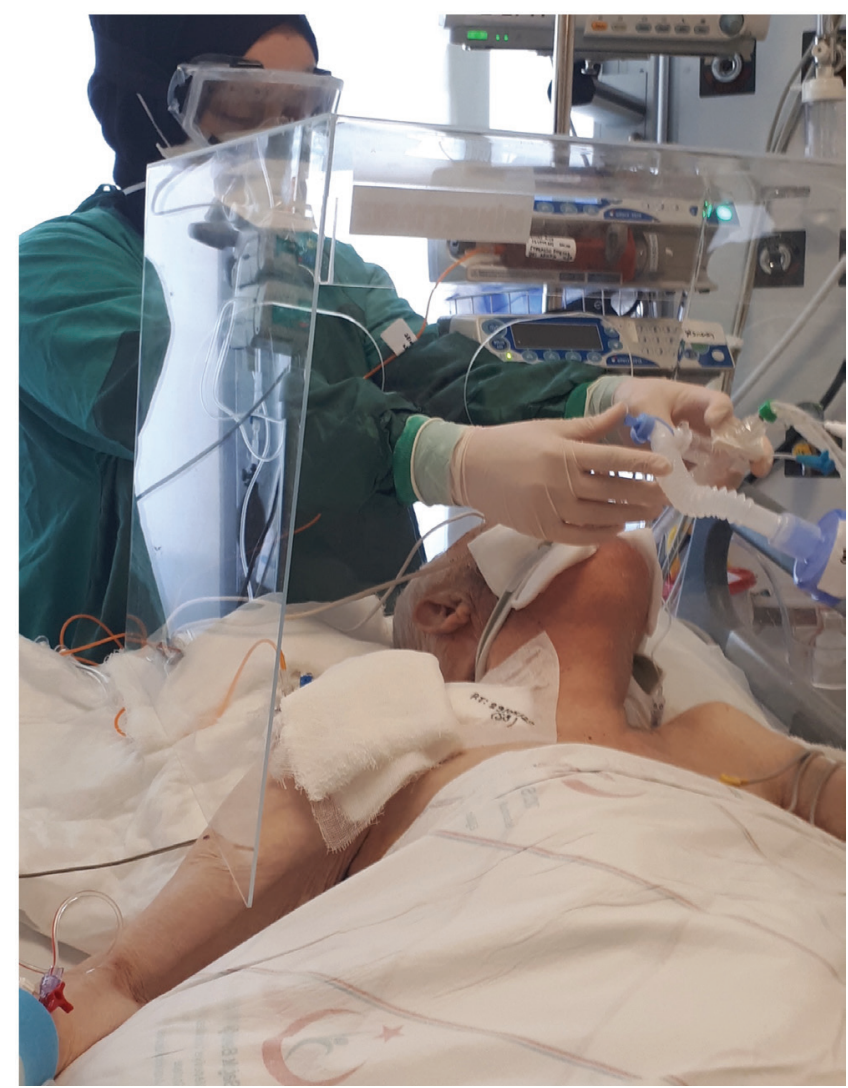

Figure 2. Protective fiberglass box during the airway intervention were smoothly performed on the first attempt. The tube cuff was inflated sufficiently to prevent the droplets from spreading with the patient's exhaled air before the ventilation was initiated.

\section{HFNC Oxygen Therapy}

We applied the HFNC treatment at a rate of 45-60 L/ min oxygen $\left[2 /\right.$ fraction of inspired oxygen $\left(\mathrm{FiO}_{2}\right)$ between 0.4-0.6]. Cough is a common symptom in COVID-19 patients. Therefore, patients were allowed to wear N95 masks during HFNC oxygen therapy. Furthermore, when applying HFNC, we paid attention to the tight and correct placement of the nasal catheter in order to minimize lateral losses.

\section{Endotracheal Aspiration}

We used a closed suction system for endotracheal sputum aspiration in patients with invasive ventilation.

\section{Invasive Mechanical Ventilation}

The type of ventilators available in our ICUs was Servo-i Maquet and Engström Pro. Sterile medical gas was supplied to the ventilator from the central oxygen and air system and, there was no ventilator to use air compressor in our ICUs. 
During mechanical ventilation, aerosol particles carrying the virus discharged by the patient's exhaled air and cause pollution of the environment. The condensate which may carry viruses forms in the breathing circuit. Therefore we used a single-use two limbs ventilator circuit with two water traps. We placed virus filters on both the inspiratory and expiratory sides of the breathing circuit which was kept closed during mechanical ventilation. We did not replace the ventilator circuits without any obvious pollution. On the other hand, virus filters were replaced daily. The water accumulated in the water traps as a result of condensation was discharged into a cup containing disinfectant. During the replacement of the ventilator circuit and filters and, discharging of the water traps mechanical ventilator was turned off for eliminating the pollution of the environment by the patient's exhaled air.

\section{Weaning and Extubation}

Spontaneous breathing trial with T-tube has the risk of aerosols spread and lower success rate of extubation than the pressure support ventilation (PSV) for weaning (12). Therefore, we used to used PSV and continuous positive airway pressure modes for the gradual decrease of withdrawal from ventilatory support during the weaning from mechanical ventilation.

Cough and sputum may occur frequently during the removal of the tracheal tube. We placed an aspiration catheter into the mouth to remove secretions before extubation. To prevent the droplets from spreading, we used a protective box, which was sprayed with a disinfectant on its inner surface during the tracheal extubation, and covered the patient with a disposable bedspread. After extubation, we applied HFNC as described above.

\section{Other treatment procedures}

Procedures requiring intensive contact with the COVID-19 patient including urinary catheterization, placement of the nasogastric catheter, central venous catheterization, and other vascular procedures were carried out using PPE as in respiratory interventions.

Hydroxychloroquine has in vitro activity against the SARS-CoV-2 virus and it decreases viral load in nasopharyngeal swabs. Its antiviral effects may be due to inhibition of virus entry and immune modulation (1315). Therefore, hydroxychloroquine was considered as a potential drug for the treatment of COVID-19 at the onset of the pandemic (16). Nevertheless, in a systematic review and meta-analysis, it has been reported that hydroxychloroquine does not improve clinical outcomes in COVID-19 (17). Moreover, it has been demonstrated that hydroxychloroquine was not useful as postexposure prophylaxis for COVID-19 (18). However, whether pre- exposure prophylaxis would be beneficial in high-risk populations is a distinct question, with trials ongoing. In vitro, it has been showing that hydroxychloroquine may be useful as a prophylactic agent against the SARS-CoV-2 virus especially for high-risk populations such as healthcare workers (19). In our hospital, all ICU workers received hydroxychloroquine phosphate (Plaquenil 200 mg, Sanofi Aventis, Turkey) $400 \mathrm{mg}$ two times a week. We didn't observe any side effects related to hydroxychloroquine phosphate.

\section{Study Limitations}

There were several limitations to the present study. The retrospective nature of the study was the first one. Failure to consider other factors such as demographic characteristics, the existence of the concomitant disease, and medical treatment of ICU workers were the other limitations of the study.

\section{Conclusion}

Current data showed that approximately $12-17 \%$ of COVID-19 patients developed severe pneumonia requiring respiratory support treatment in the $\operatorname{ICU}(5,6)$. To the best of our knowledge theretofore it has not been reported the incidence of COVID-19 among ICU workers in the literature. Despite the high risk of contagion during the aerosol-generating respiratory interventions and the other procedures requiring close contact to the patient, the incidence of COVID-19 for our ICUs and IMCU workers was very low. Our experience showed that the correct and proper use of PPE according to the protocols is effective in protecting ICU workers against the contagion of SARS$\mathrm{CoV}-2$. Moreover informing and educating of healthcare professionals about the disease process and the use of PPE was also important. Although there is no evidence, prophylaxis with hydroxychloroquine may also have been useful.

\section{Authorship Contributions}

Concept: S.U., T.A.A., Design: S.U., T.A.A., Data Collection or Processing: T.A.A., Analysis or Interpretation: S.U., T.A.A., Literature Search: S.U., T.A.A., Writing: S.U.

Conflict of Interest: No conflict of interest was declared by the authors.

Financial Disclosure: The authors declared that this study received no financial support.

\section{References}

1. Demirbilek Y, Pehlivantürk G, Özgüler ZÖ, Alp Meşe E. COVID-19 outbreak control, example of ministry of health of Turkey. Turk J Med Sci 2020;50:489-94.

2. Epidemiology Working Group for NCIP Epidemic Response, Chinese Center for Disease Control and Prevention. [The epidemiological characteristics of an outbreak of 2019 novel 
coronavirus diseases (COVID-19) in China]. Zhonghua Liu Xing Bing Xue Za Zhi 2020:145-51.

3. Su A. Doctors and Nurses Fighting Coronavirus in China Die of Both Infection and Fatigue. Los Angeles Times [online]. 2020; february 25 Available at: https://www.latimes. com/world-nation/story/2020-02-25/doctors-fightingcoronavirus-in china-die-of-both-infection-and-fatigue. Accessed March 20, 2020.

4. Euronews. https://tr.euronews.com/2020/04/29/sagl-kbakan-koca-covid-19-la-mucadelede-gelinen-son-noktaydegerlendiriyor

5. Chen N, Zhou M, Dong X, et al. Epidemiological and clinical characteristics of 99 cases of 2019 novel coronavirus pneumonia in Wuhan, China: a descriptive study. Lancet 2020;395:507-13.

6. Huang C, Wang $Y$, Li X, et al. Clinical features of patients infected with 2019 novel coronavirus in Wuhan, China. Lancet 2020;395:497-506.

7. Wang J, Zhou M, Liu F. Reasons for healthcare workers becoming infected with novel coronavirus disease 2019 (COVID-19) in China. J Hosp Infect 2020;105:100-1.

8. Schwartz J, King CC, Yen MY. Protecting Health Care Workers during the COVID-19 Coronavirus Outbreak -Lessons from Taiwan's SARS response [published online ahead of print, 2020 Mar 12]. Clin Infect Dis 2020;255.

9. Respiratory care committee of Chinese Thoracic Society. [Expert consensus on preventing nosocomial transmission during respiratory care for critically ill patients infected by 2019 novel coronavirus pneumonia]. Zhonghua Jie He He Hu Xi Za Zhin 2020;17:20.

10. Ferioli M, Cisternino C, Leo V, Pisani L, Palange P, Nava S. Protecting healthcare workers from SARS-CoV-2 infection: practical indications. Eur Respir Rev 2020;29:200068.
11. Liang T Prevention and control management. In: Handbook of COVID-19 prevention and treatment. The First Affiliated Hospital, Zhejiang University School of Medicine. Compiled According to Clinical Experience. Liang et al (eds). Zhejiang University School of Medicine, Zheijang 2020;1-18.

12. Ladeira MT, Vital FM, Andriolo RB, Andriolo BN, Atallah AN, Peccin MS. Pressure support versus T-tube for weaning from mechanical ventilation in adults. Cochrane Database Syst Rev 2014;2014:CD006056.

13. Yao $X$, Ye F, Zhang $M$, et al. In Vitro Antiviral Activity and Projection of Optimized Dosing Design of Hydroxychloroquine for the Treatment of Severe Acute Respiratory Syndrome Coronavirus 2 (SARS-CoV-2). Clin Infect Dis 2020;71732-9.

14. Gautret P, Lagier JC, Parola P, et al. Hydroxychloroquine and azithromycin as a treatment of COVID-19: results of an open-label non-randomized clinical trial. Int J Antimicrob Agents 2020;56:105949.

15. Sun $X, N i$ Y, Zhang M. Rheumotologitsts' view on the use of hydroxychloroquine to treat COVID-19. Emerg Microbes Infect 2020;9:830-2.

16. Sinha N, Balayla G. Hydroxychloroquine and COVID-19. Postgrad Med J 2020;96:550-5.

17. Elavarasi A, Prasad M, Seth $T$, et al. Chloroquine and Hydroxychloroquine for the Treatment of COVID-19: a Systematic Review and Meta-analysis. J Gen Intern Med 2020;35:3308-14.

18. Boulware DR, Pullen MF, Bangdiwala AS, et al. A Randomized Trial of Hydroxychloroquine as Postexposure Prophylaxis for Covid-19. N Engl J Med 2020;383:517-25.

19. Al-Kofahi M, Jacobson P, Boulware DR, et al. Finding the Dose for Hydroxychloroquine Prophylaxis for COVID-19: The Desperate Search for Effectiveness. Clin Pharmacol Ther 2020;108:766-9. 